\title{
The Quantum Effect on Friedmann Equation in FRW Universe
}

\author{
Wei Zhang ${ }^{1}$ and Xiao-Mei Kuang $\mathbb{D}^{2}$ \\ ${ }^{1}$ Department of General Studies, Nanchang Institute of Science \& Technology, Nanchang 330108, China \\ ${ }^{2}$ Center for Gravitation and Cosmology, College of Physical Science and Technology, Yangzhou University, Yangzhou 225009, China
}

Correspondence should be addressed to Xiao-Mei Kuang; xmeikuang@gmail.com

Received 2 December 2017; Revised 4 February 2018; Accepted 5 February 2018; Published 4 March 2018

Academic Editor: Izzet Sakalli

Copyright (C) 2018 Wei Zhang and Xiao-Mei Kuang. This is an open access article distributed under the Creative Commons Attribution License, which permits unrestricted use, distribution, and reproduction in any medium, provided the original work is properly cited. The publication of this article was funded by SCOAP ${ }^{3}$.

\begin{abstract}
We study the modified Friedmann equation in the Friedmann-Robertson-Walker universe with quantum effect. Our modified results mainly stem from the new entropy-area relation and the novel idea of Padmanabhan, who considers the cosmic space to be emerging as the cosmic time progresses, so that the expansion rate of the universe is determined by the difference of degrees of freedom between the holographic surface and the bulk inside. We also discuss the possibility of having bounce cosmological solution from the modified Friedmann equation in spatially flat geometry.
\end{abstract}

\section{Introduction}

In the 1970s, the thermodynamic property of black holes has been proposed [1-3], and it reveals that the gravitational dynamics is entwined with thermodynamics. Inspired by Bekenstein's entropy-area theorem [1], Bardeen et al. put forward the four thermodynamical laws of black hole systems [2]. In 1995, Jacobson considered Einstein's field equation as an equation of state. Afterwards, he reproduced Einstein's field equation by demanding that the fundamental relation $\delta Q=T d S$ holds for all local Rindler causal horizons through each space-time point, and $\delta Q$ and $T$ are treated as the energy flux and Unruh temperature, respectively, felt by an accelerated observer inside the horizon [4]. In 2010, Verlinde defined gravity as an entropic force due to the changes of the information related to the positions of the materials, and the space is emergent based on the holographic principle in his discussions [5]. Moreover, Verlinde's proposal has been applied to reproduce the Friedmann equation into brane cosmology [6] and Friedmann-Robertson-Walker (FRW) universe [7], respectively.

On the other hand, it was addressed in [8-12] that the Friedmann equation can be modified by a bounce solution of the universe as

$$
H^{2}=\frac{8 \pi G}{3} \rho\left(1-\frac{\rho}{\rho_{c}}\right)
$$

in loop quantum cosmology (LQC). Then the authors of [13] attempted to derive the Friedmann equation by borrowing the Clausius relation, that is, $\delta Q=T d S$, and an entropyarea relation with quantum correction; however, they failed to reproduce the same modified Friedmann equation as that in LQC with bounce solution. Luckily, the difficulty was overcome by the authors of [14], where they proposed a modified dispersion relation at quantum phenomenological level and then obtained the modified Friedmann equation for a bounce solution to the flat FRW universe in LQC. It was found that the role of their modified dispersion relation is to explicitly modify the Clausius relation. This proposal has also been extended into the spatially curved cases and the corresponding modified entropy-area relations have been derived [15].

However, the above studies are only involved in the gravity as an emergent phenomenon rather than the spacetime itself as an emergent structure. This situation has been improved by Padmanabhan. In detail, he proposed in [16] that the accelerated expansion of the universe is related to the difference between the surface degrees of freedom $\left(N_{\text {sur }}\right)$ and the bulk degrees of freedom $\left(N_{\text {bulk }}\right)$ in a region of space; that is, $\Delta V=\Delta t\left(N_{\text {sur }}-N_{\text {bulk }}\right)$, where $V$ is the Hubble volume and $t$ is the cosmic time in Planck units. Moreover, the standard evolution of the universe was also reproduced directly from the proposed relation. This proposal inspired 
plenty of related studies and remarkable progress [17-33]. However, in the framework of Padmanabhan's conjecture, the study of quantum effect is missing. So, it is interesting to introduce the quantum effect to Padmanabhan's conjecture and study the related cosmology.

Thus, in this paper, we will introduce the modified dispersion relation in the framework of Padmanabhan's conjecture and derive the modified Friedmann equation. Then we will analyze whether the quantum effect in Padmanabhan's conjecture will bring in modified Friedmann equation (1) with bounce solution. It is notable that starting from the Clausius relation to the apparent horizon along with the modified dispersion relation, one can easily get the modified Friedmann equation with bounce solution to the FRW universe $[14,15]$, but the answer is not direct in Padmanabhan's conjecture in the emergent universe. Our study will give an insight into the answer.

Our paper is organized as follows. In next section we briefly review Padmanabhan's idea that the cosmic space is emergent as cosmic time progresses and give the standard Friedmann equation governing the dynamical evolution of the FRW universe. Then, in Section 3, we will analyze the modified Friedmann equation from Padmanabhan's conjecture based on modified entropy-area relation. Finally, we will give our summary and discussions in Section 4 . In this paper, we use the natural units with $c=\hbar=k_{B}=1$.

\section{The Emergence of Cosmic Space of the FRW Universe}

In this section, we will give a brief review on the process of obtaining standard Friedmann equation in the emergent universe, which was addressed by Padmanabhan in [16]. The main idea is that the expansion of the universe (or the emergence of space) tends to fulfill the holographic equipartition condition, which stated that the number of degrees of freedom $\left(N_{\text {bulk }}\right)$ inside the Hubble volume is equal to the number of degrees of freedom $\left(N_{\text {sur }}\right)$ on the spherical surface of Hubble radius; that is, $N_{\text {bulk }}=N_{\text {sur. }}$. So in our asymptotic de Sitter universe, the natural law governing the emergence of space in an infinitesimal interval $d t$ is

$$
\frac{d V}{d t}=G\left(N_{\text {sur }}-N_{\text {bulk }}\right),
$$

where $V=4 \pi / 3 H^{3}$ is the Hubble volume and $t$ is the cosmic time.

For a spatially flat FRW universe with Hubble constant $H$ and apparent horizon $r_{A}=1 / H$, we have

$$
N_{\text {sur }}=4 S=\frac{4 \pi}{G H^{2}},
$$

where $S=A / 4 G=\pi / G H^{2}$ is the entropy of the apparent horizon, and

$$
N_{\text {bulk }}=\frac{2|E|}{T}=-\frac{2(\rho+3 p) V}{T},
$$

where in the second equality we recalled the horizon temperature $T=H / 2 \pi$ and Komar energy $|E|=-(\rho+3 p) V$ for accelerating part with dark energy having $\rho+3 p<0$ (it is notable that in [16] the author discussed the contributions of the matter with $|E|=(\rho+3 p) V$ in the bulk degrees of freedom and the derivation of Friedmann equation was unaffected). Subsequently, one can reduce (2) into

$$
\frac{\ddot{a}}{a}=-\frac{4 \pi G}{3}(\rho+3 p),
$$

which is the standard dynamical Friedmann equation of flat FRW universe in general relativity. Furthermore, recalling the continuity equation,

$$
\dot{\rho}+3 H(\rho+p)=0,
$$

and integrating (5) gives us the standard Friedmann equation

$$
H^{2}=\frac{8 \pi G \rho}{3} \text {. }
$$

Note that in [13] the integration result is $H^{2}+k / a^{2}=8 \pi G \rho / 3$ with general geometry, where the authors interpreted the integration constant $k$ as the spatial curvature of the FRW universe.

\section{The Quantum Effect on Friedmann Equation in Cosmic Space of the FRW Universe}

In this section, we will apply the proposal described in last section to study the quantum effect on the Friedmann equation. We only consider the quantum effect at the phenomenological level and borrow the modified dispersion relation (MDR) [14]:

$$
\frac{\sin \left(\eta l_{p} E\right)}{\eta l_{p}}=\sqrt{p^{2}+m^{2}} .
$$

Here $p$ and $E$ are the momentum and energy of a particle with mass $m$, respectively. The Planck length is $l_{p}=\sqrt{8 \pi G}=$ $1 / M_{p}$, where $M_{p}$ is the Planck mass. $\eta$ is a dimensionless parameter and $\eta \rightarrow 0$ goes to the standard dispersion relation $E^{2}=p^{2}+m^{2}$.

With the use of thermodynamical description on the apparent horizon, the authors of [14] derived the modified Friedmann equation of a spatially flat universe from MDR (8). Later, the extended study in general FRW universe with $k=0, \pm 1$ was presented in [15].

Here, we will derive the modified Friedmann equation by following the steps of emergent cosmic space shown in last section. According to the study in [15], MDR (8) modified the entropy for the first energy branch as

$$
\begin{aligned}
S_{M}= & \frac{A}{4 G} \sqrt{1-\frac{4 \pi \eta^{2} l_{p}^{2}}{A}} \\
& +\frac{\pi \eta^{2} l_{p}^{2}}{G} \ln \left[\sqrt{\frac{A}{4 \pi \eta^{2} l_{p}^{2}}}+\sqrt{\frac{A}{4 \pi \eta^{2} l_{p}^{2}}-1}\right],
\end{aligned}
$$


where $A=4 \pi r_{A}^{2}=4 \pi /\left(H^{2}+k / a^{2}\right)$ is the area of the apparent horizon at the classical level. To proceed, we define an effective apparent horizon area with the quantum effect

$$
\begin{aligned}
\widetilde{A}= & 4 G S_{M} \\
= & A \sqrt{1-\frac{4 \pi \eta^{2} l_{p}^{2}}{A}} \\
& +4 \pi \eta^{2} l_{p}^{2} \ln \left[\sqrt{\frac{A}{4 \pi \eta^{2} l_{p}^{2}}}+\sqrt{\frac{A}{4 \pi \eta^{2} l_{p}^{2}}-1}\right] \\
= & 4 \pi r_{A}^{2} \sqrt{1-\frac{\eta^{2} l_{p}^{2}}{r_{A}^{2}}} \\
& +4 \pi \eta^{2} l_{p}^{2} \ln \left[\frac{r_{A}}{\eta l_{p}}+\sqrt{\frac{r_{A}^{2}}{\eta^{2} l_{p}^{2}}-1}\right] .
\end{aligned}
$$

Note that when $\eta \rightarrow 0, \widetilde{A}$ is equal to $A$ and recovers the usual result.

Moreover, the volume $(V)$ and the area $(A)$ of the apparent horizon of an $n$-sphere with radius $r_{A}$ satisfy [34]

$$
\frac{d V}{d A}=\frac{r_{A}}{n-1} .
$$

Then one can think that the change of the effective volume mainly stems from the change of the effective area, so that we have the time evolution of the effective volume of the FRW universe [34]

$$
\frac{d \widetilde{V}}{d t}=\frac{r_{A}}{2} \frac{d \widetilde{A}}{d t}=\frac{4 \pi r_{A}^{3} \dot{r}_{A}}{\eta l_{p} \sqrt{r_{A}^{2} / \eta^{2} l_{p}^{2}-1}},
$$

from which we can obtain the effective volume

$$
\widetilde{V}=\frac{4 \pi \eta^{3} l_{p}^{3}}{3}\left(2+\frac{r_{A}^{2}}{\eta^{2} l_{p}^{2}}\right) \sqrt{\frac{r_{A}^{2}}{\eta^{2} l_{p}^{2}}-1 .}
$$

Also, when $\eta \rightarrow 0, \widetilde{V}=4 \pi r_{A}^{3} / 3$ is the usual Hubble volume.

We move on to calculate $N_{\text {bulk }}$ in the bulk and $N_{\text {sur }}$ in the boundary. Considering the Hawking temperature (similar to (12), we ignore the direct correction to the radius in the Hawking temperature, and the changes of numbers of degrees of freedom directly stem from the corrections of the area of the apparent horizon) $T=1 / 2 \pi r_{A}$ and $E=-(\rho+3 p) \widetilde{V}$ with dark energy in the bulk, we obtain

$$
\begin{aligned}
N_{\text {bulk }} & =\frac{2 E}{T} \\
& =-\frac{16 \pi^{2}(\rho+3 p) \eta^{3} l_{p}^{3} r_{A}}{3}\left(2+\frac{r_{A}^{2}}{\eta^{2} l_{p}^{2}}\right) \sqrt{\frac{r_{A}^{2}}{\eta^{2} l_{p}^{2}}-1 .}
\end{aligned}
$$

The statistical physics has shown that $N_{\text {sur }}$ can be calculated from the entropy [18]

$$
\begin{aligned}
N_{\text {sur }}= & 4 S_{M} \\
= & \frac{4 \pi r_{A}^{2}}{G} \sqrt{1-\frac{\eta^{2} l_{p}^{2}}{r_{A}^{2}}} \\
& +\frac{4 \pi \eta^{2} l_{p}^{2}}{G} \ln \left[\frac{r_{A}}{\eta l_{p}}+\sqrt{\frac{r_{A}^{2}}{\eta^{2} l_{p}^{2}}-1}\right] .
\end{aligned}
$$

Substituting (12), (14), and (15) into (2), we get

$$
\begin{aligned}
& \frac{4 \pi r_{A}^{3} \dot{r}_{A}}{\eta l_{p} \sqrt{r_{A}^{2} / \eta^{2} l_{p}^{2}-1}} \\
& =4 \pi r_{A}^{2} \sqrt{1-\frac{\eta^{2} l_{p}^{2}}{r_{A}^{2}}}+4 \pi \eta^{2} l_{p}^{2} \ln \left[\frac{r_{A}}{\eta l_{p}}+\sqrt{\frac{r_{A}^{2}}{\eta^{2} l_{p}^{2}}-1}\right] \\
& \quad+\frac{16 \pi^{2} G(\rho+3 p) \eta^{3} l_{p}^{3} r_{A}}{3}\left(2+\frac{r_{A}^{2}}{\eta^{2} l_{p}^{2}}\right) \sqrt{\frac{r_{A}^{2}}{\eta^{2} l_{p}^{2}}-1 .}
\end{aligned}
$$

The expression above looks very complicated; however, with $k=0$, we have $\dot{r}_{A}=1-(\ddot{a} / a) r_{A}^{2}$, so that (16) can be reduced into (with $k= \pm 1$, we have $\dot{r}_{A}=1-\left(\left(H \dot{H}-k \dot{a} / a^{3}+\right.\right.$ $\left.\sqrt{\left.\left(H^{2}+k / a^{2}\right)^{3}\right)} / \sqrt{\left(H^{2}+k / a^{2}\right)}\right) r_{A}^{2}$ which makes it difficult to simplify (16); we hope to solve this problem in near future)

$$
\begin{aligned}
\frac{\ddot{a}}{a}= & -\frac{4 \pi G(\rho+3 p)}{3}\left(1+\frac{\eta^{2} l_{p}^{2}}{r_{A}^{2}}-\frac{2 \eta^{4} l_{p}^{4}}{r_{A}^{4}}\right) \\
& +\frac{\eta^{2} l_{p}^{2}\left[2+\ln \left(\eta^{2} l_{p}^{2} / 4 r_{A}^{2}\right)\right]}{2 r_{A}^{4}} \\
\simeq & -\frac{4 \pi G(\rho+3 p)}{3}\left(1+\frac{\eta^{2} l_{p}^{2}}{r_{A}^{2}}\right)+\frac{\eta^{2} l_{p}^{2}}{r_{A}^{4}}
\end{aligned}
$$

where, in the third line, we have approximately expanded the expression to the order $\eta^{2} l_{p}^{2} / r_{A}^{2}$ because it is a small quantity. Form (17) is the modified dynamical Friedmann equation for the flat FRW universe, which reduces to the standard dynamical Friedmann equation when $\eta \rightarrow 0$. Further combining continuity equation (6) with (17), we obtain the other modified Friedmann equation

$$
\begin{aligned}
\frac{\dot{a}^{2}}{a^{2}}= & \frac{8 \pi G \rho}{3}+\frac{8 \pi G \eta^{2} l_{p}^{2}}{3 a^{2}} \int\left(\dot{a}^{2} \dot{\rho}+\frac{2 \dot{a}^{3} \rho}{a}\right) d t \\
& +\frac{2 \eta^{2} l_{p}^{2}}{a^{2}} \int \frac{\dot{a}^{5}}{a^{3}} d t .
\end{aligned}
$$

Here we also set the integration constant to be vanished. Again, $\eta \rightarrow 0$ in (18) reproduces the standard result of flat FRW universe. 
In order to analyze whether (18) admits a bounce solution, we define

$$
\rho_{c}=-\frac{\rho^{2}}{\left(\eta^{2} l_{p}^{2} / a^{2}\right) \int\left(\dot{a}^{2} \dot{\rho}+2 \dot{a}^{3} \rho / a\right) d t+\left(3 \eta^{2} l_{p}^{2} / 4 \pi G \rho a^{2}\right) \int\left(\dot{a}^{5} / a^{3}\right) d t},
$$

so that (18) can be rewritten as (1) for bounce solution. The unsolved integral in $\rho_{c}$ makes it difficult to give a reliable conclusion; however, we can at least give some discussions. First, without the quantum correction, that is, $\eta \rightarrow 0, \rho_{c}$ goes to infinity, so bounce case (1) recovers standard case (7) without bounce. Then, when $\rho_{c}$ in (19) is positive, (17) and (18) fulfill the bouncing conditions, that is, $a>0$, $\dot{a}=0$, and $\ddot{a}>0$; then (18) admits a bounce solution. Finally, when $\rho_{c}$ is nonpositive, we can not have any bounce solution.

We note that, for the second energy branch whose entropy is $-S_{M}$ [15], the procedures above are straightforward and the modified Friedmann equation is the same as (17) and (18). However, for this energy branch, the effective volume $(\widetilde{V})$ and the area $(\widetilde{A})$ of the apparent horizon are negative, which are not physical.

\section{Summary and Discussions}

In this paper, we studied the quantum effect on the Friedmann equation for the flat FRW universe with the use of Padmanabhan's conjecture in the emergent universe. We obtained modified Friedmann equations (17) and (18) with the quantum correction on dispersion relation (8). For the closed $(k=1)$ and open $(k=-1)$ universes, we found it is difficult to simplify the dynamical equation in our process, but we still see the quantum effect on (16) which is supposed to be the modified Friedmann equation. We also argued the condition under which modified Friedmann equation (18) admits a bounce solution in the flat universe.

It is worth pointing out that, in our paper, the modified Friedmann equation was only obtained in the flat FRW universe with $k=0$, which may imply that key equation (2) is not the basic equation of the emergent universe and it may have to be corrected at the quantum level. This is an interesting point we will study in the near future. On the other hand, the experimental testing of quantum effect in bouncing cosmology is another interesting aspect. There are many literatures discussed this topic, for example, [35, 36] comparing the quantum theory with experimental data and $[37,38]$ probing the quantum gravity with modified dispersion relation by cold atoms.

\section{Conflicts of Interest}

The authors declare that they have no conflicts of interest.

\section{Acknowledgments}

The authors appreciate Yi Ling and Wen-Jian Pan for helpful discussion. This work is supported by the National Natural
Science Foundation of China under Grant no. 11705161 and Natural Science Foundation of Jiangsu Province under Grant no. BK20170481.

\section{References}

[1] J. D. Bekenstein, "Black holes and entropy," Physical Review D: Particles, Fields, Gravitation and Cosmology, vol. 7, pp. 23332346, 1973.

[2] J. M. Bardeen, B. Carter, and S. W. Hawking, "The four laws of black hole mechanics," Communications in Mathematical Physics, vol. 31, pp. 161-170, 1973.

[3] S. W. Hawking, "Particle creation by black holes," Communications in Mathematical Physics, vol. 43, no. 3, pp. 199-220, 1975.

[4] T. Jacobson, "Thermodynamics of spacetime: the Einstein equation of state," Physical Review Letters, vol. 75, no. 7, pp. 1260-1263, 1995.

[5] E. Verlinde, "On the origin of gravity and the laws of Newton," Journal of High Energy Physics, vol. 29, 2011.

[6] Y. Ling and J. Wu, "A note on entropic force and brane cosmology," Journal of Cosmology and Astroparticle Physics, vol. 2010, no. 08, article 017, 2010.

[7] R.-G. Cai, L.-M. Cao, and N. Ohta, "Friedmann equations from entropic force," Physical Review D: Particles, Fields, Gravitation and Cosmology, vol. 81, Article ID 061501, 2010.

[8] M. Bojowald, "Loop quantum cosmology," Living Reviews in Relativity, vol. 8, no. 11, 2005.

[9] A. Ashtekar, T. Pawlowski, and P. Singh, "Quantum nature of the big bang," Physical Review Letters, vol. 96, no. 14, Article ID 141301, 2006.

[10] A. Ashtekar, T. Pawlowski, and P. Singh, "Quantum nature of the big bang: Improved dynamics," Physical Review D: Particles, Fields, Gravitation and Cosmology, vol. 74, no. 8, Article ID 084003, 2006.

[11] A. Ashtekar, T. Pawlowski, P. Singh, and K. Vandersloot, "Loop quantum cosmology of k=1 FRW models," Physical Review D: Particles, Fields, Gravitation and Cosmology, vol. 75, no. 2, Article ID 024035, 2007.

[12] A. Ashtekar and E. Wilson-Ewing, "Covariant entropy bound and loop quantum cosmology," Physical Review D: Particles, Fields, Gravitation and Cosmology, vol. 78, no. 6, Article ID 064047, 2008.

[13] R.-G. Cai, L.-M. Cao, and Y.-P. Hu, "Corrected entropy-area relation and modified Friedmann equations," Journal of High Energy Physics, vol. 2008, 2008.

[14] Y. Ling, W. Li, and J. Wu, "Bouncing universe from a modified dispersion relation," Journal of Cosmology and Astroparticle Physics, vol. 2009, no. 11, pp. 016-016, 2009.

[15] W.-J. Pan and Y.-C. Huang, "Bouncing universe with modified dispersion relation," General Relativity and Gravitation, vol. 48, no. $144,2016$. 
[16] T. Padmanabhan, "Emergence and Expansion of Cosmic Space as due to the Quest for Holographic Equipartition," https://arxiv.org/abs/1206.4916.

[17] K. Yang, Y. Liu, and Y. Wang, "Emergence of cosmic space and the generalized holographic equipartition," Physical Review D: Particles, Fields, Gravitation and Cosmology, vol. 86, no. 10, 2012.

[18] F.-Q. Tu and Y.-X. Chen, "Emergence of spaces and the dynamic equations of FRW universes in the $\mathrm{f}(\mathrm{R})$ theory and deformed Horava-Lifshitz theory," Journal of Cosmology and Astroparticle Physics, vol. 1305, no. 024, 2013.

[19] Y. Ling and W. Pan, "Note on the emergence of cosmic space in modified gravities," Physical Review D: Particles, Fields, Gravitation and Cosmology, vol. 88, no. 4, 2013.

[20] M. Eune and W. Kim, "Emergent Friedmann equation from the evolution of cosmic space revisited," Physical Review D: Particles, Fields, Gravitation and Cosmology, vol. 88, no. 6, article 067303, 2013.

[21] F.-Q. Tu and Y.-X. Chen, "Emergence of space and cosmic evolution based on entropic force," General Relativity and Gravitation, vol. 47, no. 8, 2015.

[22] R.-G. Cai, "Emergence of space and spacetime dynamics of Friedmann-Robertson-Walker universe," Journal of High Energy Physics, vol. 88, no. 8, article 084019, 2012.

[23] M. Hashemi, S. Jalalzadeh, and S. Vasheghani Farahani, "Hawking temperature and the emergent cosmic space," General Relativity and Gravitation, vol. 47, no. 4, 2015.

[24] A. Sheykhi, M. H. Dehghani, and S. E. Hosseini, "Friedmann equations in braneworld scenarios from emergence of cosmic space," Physics Letters B, vol. 726, no. 1-3, pp. 23-27, 2013.

[25] C.-Y. Ee and D. Lee, "Friedmann equation and the emergence of cosmic space," Journal of High Energy Physics, vol. 1404, no. 125, 2014.

[26] A. Sheykhi, M. H. Dehghani, and S. E. Hosseini, "Emergence of spacetime dynamics in entropy corrected and braneworld models," Journal of Cosmology and Astroparticle Physics, vol. 2013, 2013.

[27] A. Sepehri, F. Rahaman, A. Pradhan, and I. H. Sardar, "Emergence and expansion of cosmic space in BIonic system," Physics Letters B, vol. 741, pp. 92-96, 2015.

[28] Z.-L. Wang, W.-Y. Ai, H. Chen, and J.-B. Deng, "Cosmological model from emergence of space," Physical Review D: Particles, Fields, Gravitation and Cosmology, vol. 92, no. 2, article 024051, 2015.

[29] A. Sepehri, F. Rahaman, S. Capozziello, A. F. Ali, and A. Pradhan, "Emergence and oscillation of cosmic space by joining M1-branes," The European Physical Journal C, vol. 76, no. 5, article no. 231, 2016.

[30] F.-F. Yuan and P. Huang, "Emergent cosmic space in Rastall theory," Classical and Quantum Gravity, vol. 34, no. 7, article 077001, 2017.

[31] N. Komatsu, "Cosmological model from the holographic equipartition law with a modified Rényi entropy," The European Physical Journal C, vol. 77, no. 4, 2017.

[32] P. Krishna and T. K. Mathew, "Holographic equipartition and the maximization of entropy," Physical Review D: Particles, Fields, Gravitation and Cosmology, vol. 96, no. 6, 2017.

[33] F. Q. Tu, Y. X. Chen, B. Sun, and Y. C. Yang, "Accelerated Expansion of the Universe based on Emergence of Space and Thermodynamics of the Horizon," https://arxiv.org/abs/1707.06461.

[34] R.-G. Cai, "Emergence of space and spacetime dynamics of Friedmann-Robertson-Walker universe," Journal of High Energy Physics, vol. 1211, no. 016, 2012.
[35] G. Amelino-Camelia, "Introduction to quantum-gravity phenomenology," Lecture Notes in Physics, vol. 669, no. 59, 2005.

[36] G. Amelino-Camelia, C. Lämmerzahl, A. Maclas, and H. Müller, "The search for Quantum gravity signals," in AIP Conference Proceedings, 758, 2005.

[37] G. Amelino-Camelia, C. Lämmerzahl, F. Mercati, and G. M. Tino, "Constraining the Energy-Momentum Dispersion Relation with Planck-Scale Sensitivity Using Cold Atoms," Physical Review Letters, vol. 103, article 171302, 2009.

[38] F. Mercati, D. Mazon, G. Amelino-Camelia et al., "Probing the quantum-gravity realm with slow atoms," Classical and Quantum Gravity, vol. 27, article 215003, 2010. 

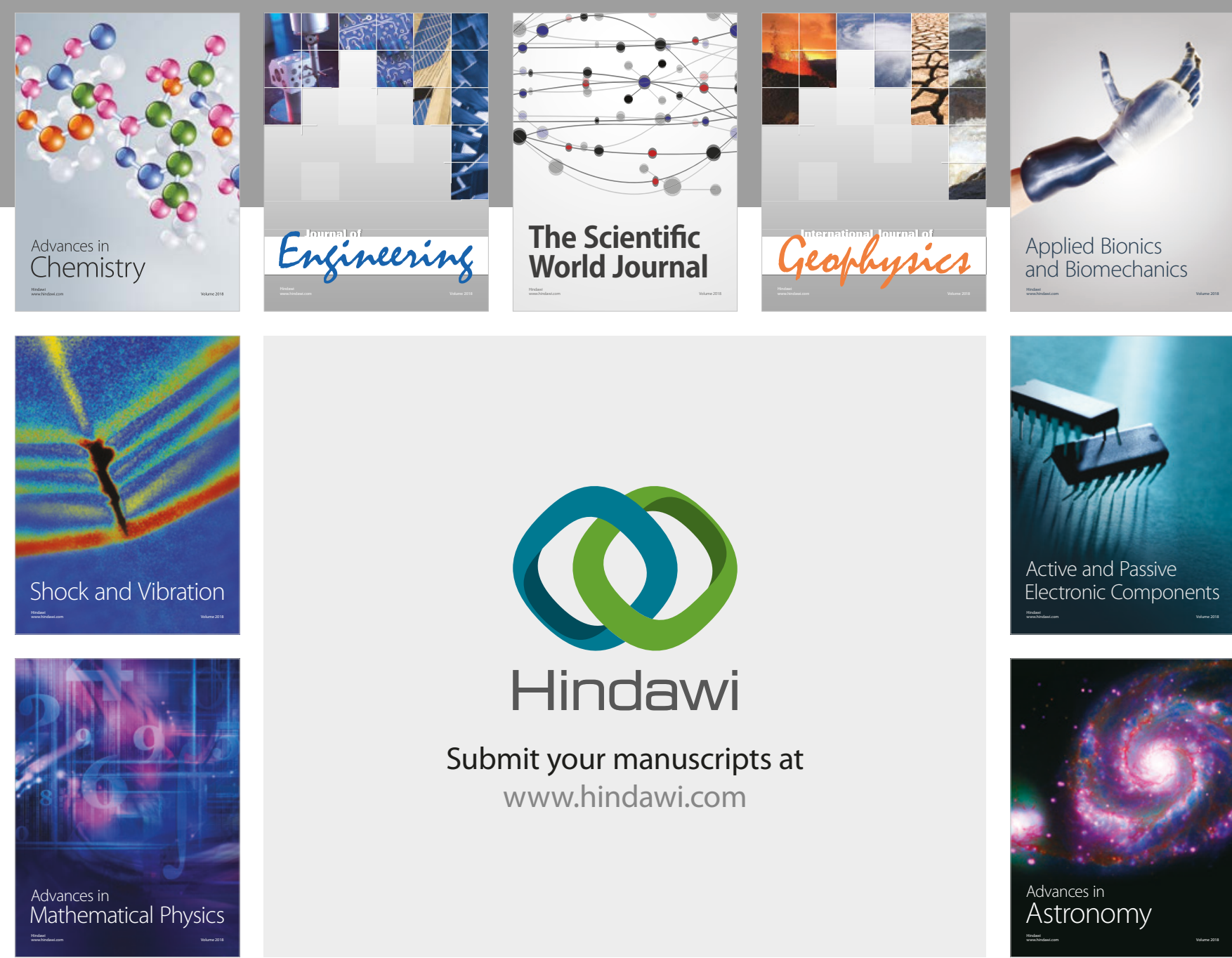

Submit your manuscripts at

www.hindawi.com

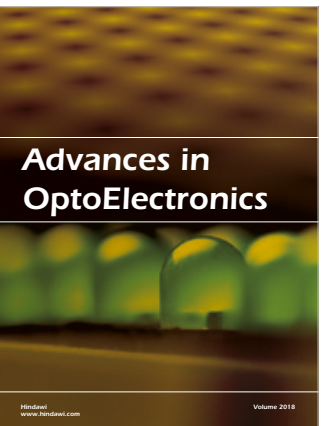

\section{Rotcting Machinery}
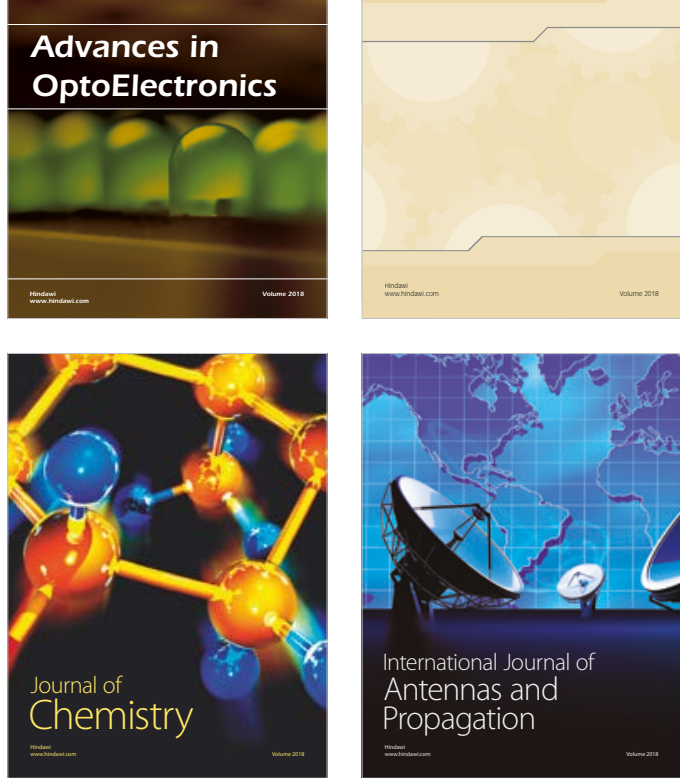

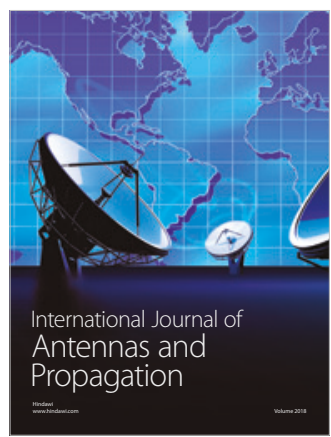

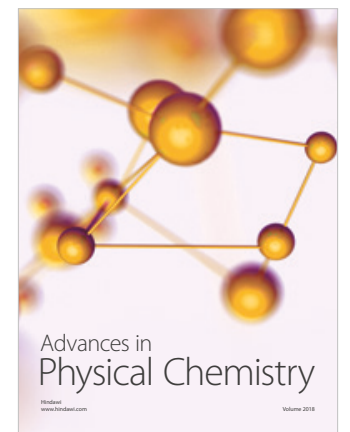

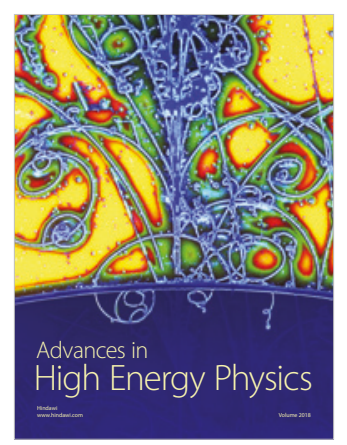

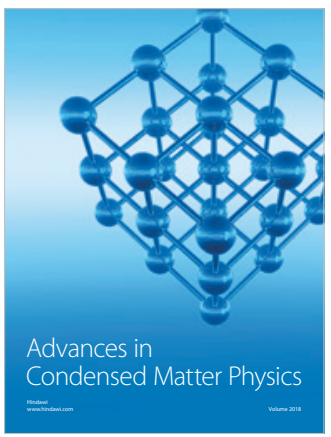

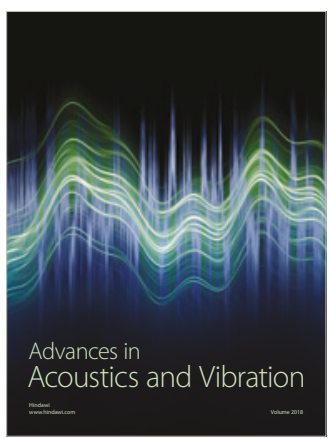

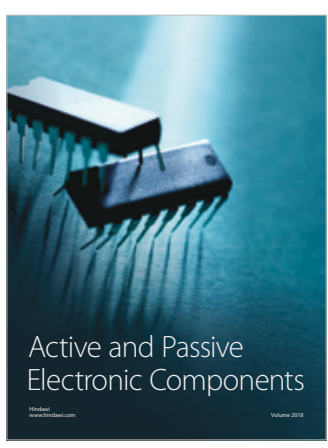
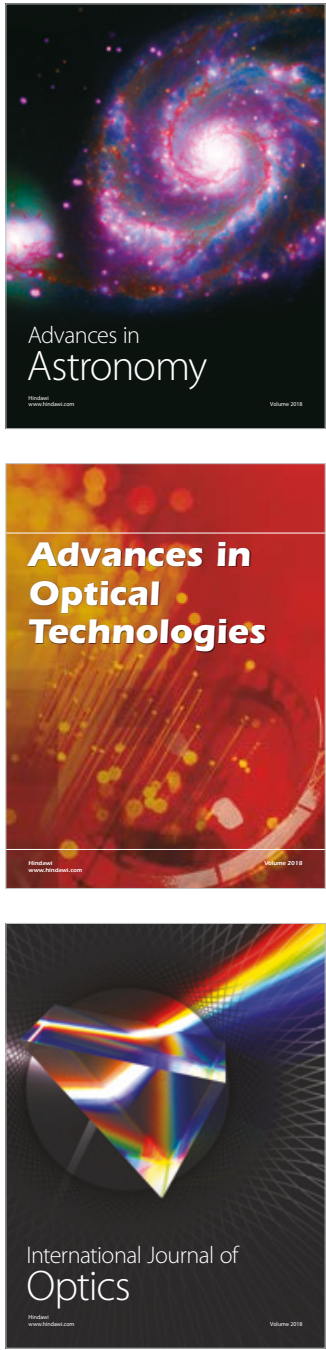